\title{
Towards the Automated Verification of Multithreaded Java Programs
}

\author{
Giorgio Delzanno ${ }^{1}$, Jean-François Raskin ${ }^{2 \star}$, and Laurent Van Begin ${ }^{2 \star \star}$ \\ 1 Dipartimento di Informatica e Scienze dell'Informazione \\ Università di Genova, via Dodecaneso 35, 16146 Genova, Italy \\ 2 Département d'Informatique, Université Libre de Bruxelles, \\ Blvd Du Triomphe, 1050 Bruxelles, Belgium
}

\begin{abstract}
In this paper we investigate the possible application of $p a$ rameterized verification techniques to synchronization skeletons of multithreaded Java programs. As conceptual contribution, we identify a class of infinite-state abstract models, called Multi-Transfer Nets (MTNs), that preserve the main features of the semantics of concurrent Java. We achieve this goal by exploiting an interesting connection with the Broadcast Protocols of [7, and by introducing the notion of asynchronous rendez-vous. As technical contribution, we extend the symbolic verification techniques of [6] based on Covering Sharing Trees and structural invariants to MTNs. As practical contribution, we report on experimental results for verification of examples of multithreaded Java programs.
\end{abstract}

\section{Introduction}

In the last years there has been an increasing interest in automated verification techniques for parameterized systems. Contrary to approaches based on finite-state abstractions, in parameterized verification it is possible to handle infinite-state abstract models, with a potential gain of precision in the analysis of the underlying systems. Recently, this idea has been applied to the verification of safety properties of multithreaded $C$ programs [2], a natural field of application for techniques related to Petri Nets. Concurrent Java, however, is going to become the standard language for multithreaded programming. Its success is due to technology like Applets and Servlets, widely used in the context of client-server applications for the World Wide Web. For this reason, we consider the specialization of parameterized verification techniques to concurrent Java programs an important (and challenging) research goal.

In this paper we will focus on problems that we think are propedeutic to further research in this direction. Specifically, we will first address the problem of finding adequate infinite-state abstract models for synchronization skeletons

\footnotetext{
* This author was partially supported by a "Crédit aux chercheurs", Belgian National Fund for Scientific Research.

** Supported by a "First Europe" grant, Walloon Region, Belgium. This work was partially done when this author was visiting Università di Genova.
} 
of concurrent Java programs. The Petri Net model adopted for C programs in [2] is no more adequate here. The problem here is due to the presence of special Java built-in methods, namely wait, notify and notifyAll, whose semantics cannot be given via rendez-vous communication. We will solve this problem by resorting to the following connections: the semantics of notifyAll (a primitive that awakens all processes waiting on a lock) can be expressed in terms of the broadcast primitive introduced by Emerson and Namjoshi in [7]; the semantics of notify (a primitive that awakens only one of the waiting processes) can be expressed via asynchronous rendez-vous, i.e., a rendez-vous that is non-blocking for the sender. We will formalize these intuitions by introducing the new model of Multi-Transfer Nets (MTNs), a formalism that incorporates and extends the main feature of Petri Nets and Broadcast Protocols.

As a second step, we will study the problem of finding an adequate technology to efficiently model check this new class of infinite-state systems. We will first show that the backward reachability algorithm of Esparza, Finkel and Mayr used for Broadcast Protocols in 8] can be naturally extended to MTNs. Decidability still holds for the control state reachability problem that consists of deciding if a state taken from a given upward closed set of unsafe states is reachable from the initial states. In [5], we have define a graph-based data structure called Covering Sharing Trees (CSTs) to compactly represent upward closed sets of markings of Petri Nets. In this paper we will show that the CST-based verification techniques defined in [6] can be extended to MTNs. Specifically, we will define a CST-based symbolic algorithm to compute the pre-image operator associated to an MTN, and we will apply it to build a symbolic backward reachability algorithm to check parameterized safety properties. In [5,6] we proposed several heuristics for the analysis of Petri Nets. Most of them are based on the Structural Theory of Petri Nets, a theory that allows to statically compute over-approximations of the reachability set. Interestingly, MTNs can be viewed as a subclass of Petri Nets with marking dependent cardinality arcs, a class of models for which Ciardo [3] an extension of the Structural Theory of Petri Nets. As a nice consequence of this connection, we can still use the pruning techniques based on structural invariants proposed in [6] to efficiently cut the search space of an MTN during backward reachability.

As practical experiments, we have applied the extended CST-library to several parameterized safety problems taken from the literature, see e.g., [7, 8. In this paper we will report on bechmarks performed over abstractions of multithreaded programs and we will compare the results with HyTech [11, a polyhedra-based model checker that provides backward reachability and that can handle the same class of parameterized systems.

\section{Abstract Models for Multithreaded Java Programs}

A Java thread is an object of the predefined Java classes Thread and Runnable. The code of a thread must be specified in the method run that is invoked to start its execution. Threads are executed in parallel and can share variables and 


\subsection{Global Machines}

Let us focus on our example and forget for a moment all synchronization primitives. Following [2, in order to extract the control skeleton of the classes Inc and Dec, we can apply the technique of predicate abstraction. We first unfold the methods of the Point class into the thread declarations. Then, we associate a boolean variable to each guard in the program (e.g. $x==0$ will be represented via a boolean variable zeroX), and extrapolate the effect of the instructions on the resulting boolean program. This way, we obtain two finite-state automata. Each state of the automata corresponds to a control point in the flattened code of the methods of our threads. Method invocations can be represented using synchronization labels. Global boolean variables can be used to model the monitor that controls a shared object.

Let us now consider the synchronization skeleton of threads. Unfortunately, communication via rendez-vous cannot be used to model the operational semantics of the interplay between the built-in methods wait, notify and notifyAll. The type of synchronization we need here involves, in fact, a number of processes that depends on the current global state of the system (all processes that are currently waiting to be awakened). To solve this problem, we will resort to a new model, called global machines, obtained by merging concepts coming from the broadcast protocols of [7] and from the global/local machines of [2] with the new notion of asynchronous rendez-vous.

We start the description of global machines from the operations needed to handle global boolean variables.

Definition 1. Let $\mathbf{B}=\left\{b_{1}, \ldots, b_{n}\right\}$ be a finite set of global boolean variables, and let $\mathbf{B}^{\prime}$ be their primed version. A boolean guard $\varphi_{g}$ is either the formula true or the conjunction of literals $L_{1} \wedge \ldots \wedge L_{p}, p \leq n$, such that $L_{i}$ is either $b$ or $\neg b$ for some $b \in \mathbf{B}$. A boolean action $\varphi_{a}$ is a formula $b_{1}^{\prime}=v_{1} \wedge \ldots \wedge b_{n}^{\prime}=v_{n}$, where $v_{i} \in\left\{\right.$ true, false, $\left.b_{i}\right\}$ for $i: 1, \ldots, n$.

Boolean guards and actions are used to express pre-and post-conditions on the variables in $\mathbf{B}$. The behaviour of a thread will be modeled via the notion of local machine introduced below.

Definition 2. A local machine is a tuple $\langle Q, \Sigma, \delta\rangle$, where: $Q$ is a finite set of states; $\Sigma$ is the set of synchronization labels used to build the set of possible actions $\mathcal{A}$ of a process (defined later); and $\delta \subseteq(Q \times \mathcal{A} \times Q)$ is the local transition relation. In the following, we will use $s \stackrel{\alpha}{\longrightarrow} s^{\prime}$ to indicate that $\left\langle s, \alpha, s^{\prime}\right\rangle \in \delta$.

The actions of a local machines are defined as follows (in the following $\varphi$ represent the conjunction of a boleean guard with an action (Def. 1), and $\ell \in \Sigma$ ):

- Internal action: $\ell: \varphi$;

- Rendez-vous: $\ell !: \varphi$ (sending), and $\ell$ ? (reception);

- Asynchronous Rendez-vous: $\ell \uparrow: \varphi$ (sending), and $\ell \downarrow$ (reception);

- Broadcast. $\ell ! ! ~: \varphi$ (sending), and $\ell$ ?? (reception). 


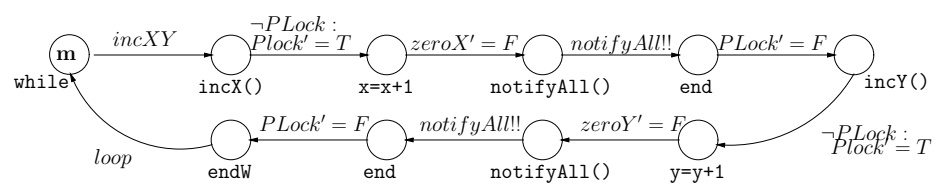

Fig. 2. The Global Machine for the Inc thread of Fig. 1

Having in mind the translation from Java programs, we will also apply the following restrictions: the set of source and target states of a broadcast (asynchronous rendez-vous) reception must be distinct; broadcasts receptions associated to the same sending can be partitioned so that each partition is defined over a distinct set of states. Note, in fact, tha we will use asynchronous rendez-vous and broadcast to model the semantics of notify and notifyAll. Our restriction avoids cyclic rules like sloc $_{1} \stackrel{n ! !}{\longrightarrow} \operatorname{sloc}_{2}$, rloc $_{1} \stackrel{n ? ?}{\longrightarrow}$ rloc $_{2}$, and $r l o c_{2} \stackrel{n ? ?}{\longrightarrow} \operatorname{rloc}_{1}$ that have no meaning if sloc and rloc are control points in the code of the sender and of the receiver, respectively, and $n$ corresponds to a notifyAll. Furthermore, all the interesting examples of Broadcast Protocols we are aware of satisfy these conditions.

As an example, the abstract model that we extracted from thread Inc applying the technique of predicate abstraction can be represented via the local machine of Fig. 2.

Definition 3. A global machine is a tuple $\mathcal{G}=\left\langle\mathbf{B},\left\langle\mathcal{L}_{1}, k_{1}\right\rangle, \ldots,\left\langle\mathcal{L}_{m}, k_{m}\right\rangle\right\rangle$, where: $\mathbf{B}=\left\{b_{1}, \ldots, b_{n}\right\}$ is the set of global boolean variables for $i: 1, \ldots, m$; $\mathcal{L}_{i}=\left\langle Q_{i}, \Sigma_{i}, \delta_{i}\right\rangle$ is the $i$-th local machine; and $k_{i}$ the number of its copies. Futhermore, we have that $Q_{i} \cap Q_{j}=\emptyset$ for any $i, j: 1, \ldots, m$ with $i \neq j$.

Definition 4. A global state of $\mathcal{G}=\left\langle\mathbf{B},\left\langle\mathcal{L}_{1}, k_{1}\right\rangle, \ldots,\left\langle\mathcal{L}_{m}, k_{m}\right\rangle\right\rangle$ is a tuple $G=$ $\langle\rho, \mathbf{s}\rangle$ where $\rho: \mathbf{B} \rightarrow\{$ true, false $\}$ is a valuation for the global boolean variables, and the tuple $\mathbf{s}=\left\langle s_{11}, \ldots, s_{1 k_{1}}, \ldots, s_{m 1}, \ldots, s_{m k_{m}}\right\rangle$ of dimension $k=k_{1}+\ldots+$ $k_{m}$ is such that $s_{i j} \in Q_{i}$ denotes the current local state of the $j$-th copy of the local machine $\mathcal{L}_{i}$.

The runs of a global machine are defined via the relation $\Rightarrow$ defined below.

Definition 5. Let $\mathcal{G}=\left\langle\mathbf{B},\left\langle\mathcal{L}_{1}, k_{1}\right\rangle, \ldots,\left\langle\mathcal{L}_{m}, k_{m}\right\rangle\right\rangle$ be a global machine, $G=$ $\left\langle\rho,\left\langle s_{1} \ldots, s_{k}\right\rangle\right\rangle$ and $G=\left\langle\rho^{\prime},\left\langle s_{1}^{\prime} \ldots, s_{k}^{\prime}\right\rangle\right\rangle$ be two global states, and $\gamma=\rho \cup \rho^{\prime}$. Then, $G \stackrel{\ell}{\Longrightarrow} \mathcal{G} G^{\prime}$ iff one of the following conditions holds:

- there exist $i$ and $u$ such that $s_{i} \stackrel{\ell: \varphi}{\longrightarrow} u$, and $\gamma(\varphi)=$ true, $s_{i}^{\prime}=u$, and $s_{j}^{\prime}=s_{j}$ for all $j \neq i$.

- there exist $i, j, u$ and $v$ such that $s_{i} \stackrel{\ell !: \varphi}{\longrightarrow} u, s_{j} \stackrel{\ell ?}{\longrightarrow} v, \gamma(\varphi)=$ true, $s_{i}^{\prime}=u$, $s_{j}^{\prime}=v$, and $s_{r}^{\prime}=s_{r}$ for all $r \neq i, j$. 
- there exist $i$ and $u$ such that $s_{i} \stackrel{\ell \uparrow: \varphi}{\longrightarrow} u$, and $\gamma(\varphi)=$ true, $s_{i}^{\prime}=u$, and: either there exist $j$ and $v$ such that $s_{j} \stackrel{\ell \downarrow}{\longrightarrow} v, s_{j}^{\prime}=v$ and $s_{r}^{\prime}=s_{r}$ for any $r \neq i, j$, or there are no $j$ and $v$ such that $s_{j} \stackrel{\ell \downarrow}{\longrightarrow} v$ is defined, and $s_{r}^{\prime}=s_{r}$ for any $r \neq i$.

- there exist $i$ and $u$ such that $s_{i} \stackrel{\ell ! ! \varphi}{\longrightarrow} u, \gamma(\varphi)=$ true, $s_{i}^{\prime}=u$, and for all $j$ such that there exist $v$ and $s_{j} \stackrel{\ell ? ?}{\longrightarrow} v$, we have $s_{j}^{\prime}=v$; finally, $s_{r}^{\prime}=s_{r}$ for all $r \neq i$ such that $\ell ?$ ? is not defined in $s_{r}$.

A run of a global machine is a sequence of global states $G_{0} G_{1} \ldots G_{n}$ such that $G_{i} \stackrel{\ell}{\Longrightarrow} G_{i+1}$ for $0 \leq i<n . G_{0}$ is the initial global state of the run and $G_{n}$ is the target global state of the run. A global state $G^{\prime}$ is reachable from $G$, written $G \stackrel{*}{\Rightarrow} \mathcal{G} G^{\prime}$, if and only if there exists a run with initial global state $G$ and target global state $G^{\prime}$. The set of reachable global states from a set of initial global states $\mathbf{G}$, noted $\operatorname{Reach}_{\mathcal{G}}(\mathbf{G})$, is equal to $\left\{G^{\prime} \mid \exists G \in \mathbf{G}: G \stackrel{*}{\Rightarrow}{ }_{\mathcal{G}} G^{\prime}\right\}$.

\section{Multi-transfer Nets}

Following [810], in order to study safety properties of global machines, we will apply a counting abstraction that maps global states into markings (of a Petri Net model) that keep track of the number of processes in each one of the local states of the local machines. To be able to model the communication mechanisms of Def. 2 we need however an extended Petri Net-like model, we will call MultiTransfer Nets (MTNs). MTNs have all the features of Petri Nets. In addition, MTNs allow us to capture the semantics of rendez-vous, asynchronous rendezvous and of broadcast as an instance of a general notion of transfer of at most $c$ tokens. Formally, this model is defined as follows.

Definition 6 (Multi-Transfer Net). A Multi-Transfer Net is a pair $\langle\mathcal{P}, \mathcal{B}\rangle$ where: $\mathcal{P}=\left\{p_{1}, \ldots, p_{n}\right\}$ is a set of places, and $\mathcal{B}=\left\{M_{1}, \ldots, M_{m}\right\}$ is a set of multi-transfers.

A multi-transfer $M$ is a tuple $\left\langle T,\left\{S_{1}, \ldots, S_{u}\right\}\right\rangle$ such that

$-T=\langle\mathcal{I}, \mathcal{O}\rangle$ is the Petri Net transition of the multi-transfer, i.e. $\mathcal{I}, \mathcal{O}: \mathcal{P} \rightarrow \mathbb{N}$;

- each $S_{k}=\left\langle\left\{B_{k 1}, \ldots, B_{k r_{k}}\right\}, c_{k}\right\rangle$ is a transfer block, where $c_{k} \in \mathbb{N} \cup\{+\infty\}$ is the bound, and each $B_{k j}=\left\langle P_{k j}, p_{k j}\right\rangle$ with $P_{k j} \subseteq \mathcal{P}$ and $p_{k j} \in \mathcal{P}$ is a transfer.

In order to avoid cyclic transfers, a multi-transfer $M$ with set of transfer blocks $\left\{S_{1}, \ldots, S_{u}\right\}$ must satisfy the following conditions:

1. for any transfer block $S_{k}$ with set of transfers $\left\{B_{k 1}, \ldots, B_{k r_{k}}\right\}$, and for any such $B_{k j}$, we require that $p_{k j} \notin P_{k j}$;

2. for any transfer $B_{k i}$ in the transfer block $S_{k}$ and $B_{l j}$ in the transfer block $S_{l}$ with $B_{k i} \neq B_{l j}$, we require that $\left(P_{k i} \cup\left\{p_{k i}\right\}\right) \cap\left(P_{l j} \cup\left\{p_{l j}\right\}\right)=\emptyset$.

A marking is a mapping $\mathbf{m}: \mathcal{P} \rightarrow \mathbb{N}$ (a vector of natural numbers). Given $\mathcal{I}$ : $\mathcal{P} \rightarrow \mathbb{N}$, we use $\mathcal{I} \geq \mathbf{m}$ to indicate that $\mathcal{I}(p) \geq \mathbf{m}(p)$ for all $p \in \mathcal{P}$. Furthermore, given $S \subseteq \mathcal{P}$ we define $\mathbf{m}(S)=\Sigma_{p \in S} \mathbf{m}(p)$. 
Definition 7 (Enabling a Multi-Transfer). Let $M$ be a multi-transfer with transition $\langle\mathcal{I}, \mathcal{O}\rangle$. We say that $M$ is enabled in $\mathbf{m}$ if $\mathcal{I} \geq \mathbf{m}$.

Definition 8 (Firing a Multi-Transfer). Let $M=\left\langle T,\left\{S_{1}, \ldots, S_{u}\right\}\right\rangle$ be a multi-transfer enabled in $\mathbf{m}$. Firing $M$ in $\mathbf{m}$ leads to any marking $\mathbf{m}^{\prime}$ (written $\mathbf{m} \succ_{M} \mathbf{m}^{\prime}$ ) computed in accord to the following sequence of steps (in which we use the two intermediate markings $\mathbf{m}_{1}$ and $\mathbf{m}_{2}$ ):

1. let $T=\langle\mathcal{I}, \mathcal{O}\rangle, \mathbf{m}_{1}(p)=\mathbf{m}(p)-\mathcal{I}(p)$ for all $p \in \mathcal{P}$;

2. $\mathbf{m}_{2}$ can be any marking such that the following constraints are satisfied:

- for each transfer block $S_{k}=\left\langle\left\{B_{k 1}, \ldots, B_{k r_{k}}\right\}, c_{k}\right\rangle$ :

- if $r_{k}>0$ and $\mathbf{m}_{1}\left(P_{k 1} \cup \ldots \cup P_{k r_{k}}\right)>c_{k}$, then for all $d_{1}, \ldots, d_{r_{k}} \in \mathbb{N}$ be such that $d_{1}+\ldots+d_{r_{k}}=c_{k}$ and for all $j, 1 \leq j \leq r_{k}$ :

- $\mathbf{m}_{2}\left(p_{k j}\right)=\mathbf{m}_{1}\left(p_{k j}\right)+d_{j}$,

- and $\mathbf{m}_{2}\left(P_{k j}\right)=\mathbf{m}_{1}\left(P_{k j}\right)-d_{j}$,

- with the additional constraint that $\mathbf{m}_{1}(p) \geq \mathbf{m}_{2}(p)$ for any $p \in$ $P_{k j}$.

- if $r_{k}>0$ and $\mathbf{m}_{1}\left(P_{k 1} \cup \ldots \cup P_{k r_{k}}\right) \leq c_{k}$, then for all $j, 1 \leq j \leq r_{k}$ :

- $\mathbf{m}_{2}\left(p_{k j}\right)=\mathbf{m}_{1}\left(p_{k j}\right)+\mathbf{m}_{1}\left(P_{k j}\right)$ and

- $\mathbf{m}_{2}\left(P_{k j}\right)=0$

- $\mathbf{m}_{2}(p)=\mathbf{m}_{1}(p)$ for all $p \in \mathcal{P}$ not involved in transfer blocks;

3. $\mathbf{m}^{\prime}(p)=\mathbf{m}_{2}(p)+\mathcal{O}(p)$ for all $p \in \mathcal{P}$.

Note that a Petri Net transition is obtained by considering multi-transfers with an empty set of transfer blocks. A transfer arc (all tokens in the sources are transferred to the target) is obtained instead by a multi-transfer with a single set of transfers with bound $c=+\infty$. A transfer block has a non-deterministic effect if the total number of tokens is strictly greater than $c$ with $c \in \mathbb{N}$. The non-determinism is due to the numbers $d_{1}, \ldots, d_{r_{k}}$ of tokens that are transferred by each transfer, and from their distribution within the set $P_{k j}$ of sources for $j: 1, \ldots, r_{k}$.

Definition 9 (Operational Semantics). Let $\mathcal{M}=\langle\mathcal{P}, \mathcal{B}\rangle$ be an MTN. A run of $\mathcal{M}$ is a sequence of markings $\mathbf{m}_{0} \mathbf{m}_{1} \ldots \mathbf{m}_{n}$ such that for any $i, 0 \leq i<n$, there exists $M \in \mathcal{B}$ such that $\mathbf{m}_{i} \longmapsto_{M} \mathbf{m}_{i+1}, \mathbf{m}_{0}$ is the initial marking of the run and $\mathbf{m}_{n}$ the target marking of the run. A marking $\mathbf{m}^{\prime}$ is reachable from a marking $\mathbf{m}$, written $\mathbf{m} \longmapsto^{*} \mathbf{m}^{\prime}$, if and only if there exists a run with initial marking $\mathbf{m}_{0}$ and target marking $\mathbf{m}^{\prime}$. The set of reachable markings of $\mathcal{M}$ from a set of markings $I$, written $\operatorname{Reach}_{\mathcal{M}}(I)$, is defined as the set $\left\{\mathbf{m}^{\prime} \mid \exists \mathbf{m} \in I: \mathbf{m} \longmapsto^{*} \mathbf{m}^{\prime}\right\}$.

\subsection{From Global Machines to MTNs}

Global machines can be naturally translated into MTNs, as we will informally explain in this section. In the following we will often refer to Fig. 3 to illustrate the intuition behind the translation. First of all, each global boolean variable $b$ is modeled in the MTN by the two places $T_{b}$ and $F_{b}$. Each local state $s_{i j}$ appearing in the local machine $\mathcal{L}_{i}$ is modelled with a place with the same name 


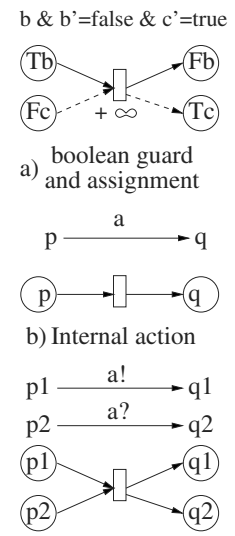

c) Rendez-vous
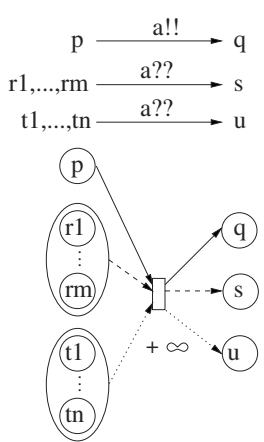

d) Broadcast

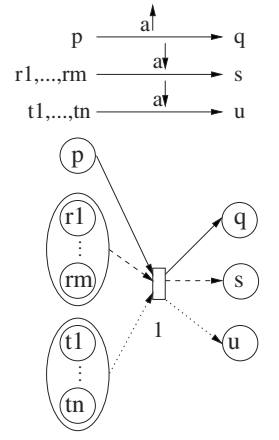

e) Asynchronous Rendez-vous

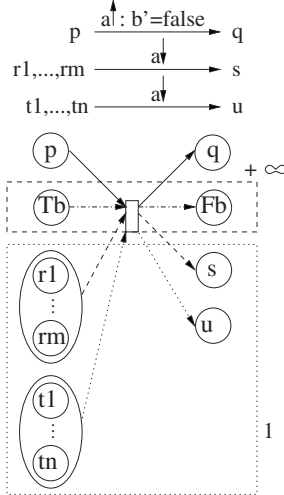

f) Complete example

Fig. 3. From global machines rules to MTNs. $r_{1}, \ldots, r_{n} \stackrel{\ell ? ?}{\longrightarrow} s$ is an abbreviation for the set of transitions $r_{1} \stackrel{\ell ? ?}{\longrightarrow} s, \ldots, r_{n} \stackrel{\ell ? ?}{\longrightarrow} s$, all of them having the same destination state $s$, similarly for $r_{1}, \ldots, r_{n} \stackrel{\ell \downarrow}{\longrightarrow} s$.

$s_{i j}$. A global state $G=\langle\rho, \mathbf{s}\rangle$ is translated into the marking $\mathbf{m}_{G}$ such that: (1) for each $b \in B$, if $\rho(b)=$ true then $\mathbf{m}_{G}\left(T_{b}\right)=1$ and $\mathbf{m}_{G}\left(F_{b}\right)=0$, if $\rho(b)=$ false then $\mathbf{m}_{G}\left(T_{b}\right)=0$ and $\mathbf{m}_{G}\left(F_{b}\right)=1 ;(2)$ for each local state $s_{i j}, \mathbf{m}_{G}\left(s_{i j}\right)=v_{i j}$, where $v_{i j}$ is the number of occurrences of state $s_{i j}$ in $\mathbf{s}$. In other words, the place $s_{i j}$ contains as many tokens as the number of copies of the local machine $\mathcal{L}_{i}$ whose current state is $s_{i j}$. Let us now briefly explain how an action is translated into a multi-transfer. We first focus our attention on the boolean part of actions. Consider the boolean formula $\varphi=b \wedge b^{\prime}=$ false $\wedge c^{\prime}=$ true with $d^{\prime}=d$ for all other variables. Let $M_{\varphi}$ be the multi-transfer that results from the translation of $\varphi$. Intuitively, $M_{\varphi}$ has to check the presence of one token in place $T_{b}$, and ensure that, when the transition is taken, the token is removed from $T_{b}$ and added to $F_{b}$. Furthermore, the only token shared by $T_{c}$ and $F_{c}$ must be in $F_{c}$ after the firing. This can be achieved as shown in Fig. [3(a). Using the Petri Net transition of $M_{\varphi}$ (thick lines in in Fig. (3), we ensure the presence of the token in $T_{b}$ before the firing and in $F_{b}$ after. To ensure the presence of a token in $T_{c}$ after firing, we use an unbounded transfer block with a single transfer from the singleton $\left\{F_{c}\right\}$ to the place $T_{c}$ (dashed lines in Fig. 31). If the token was already in $T_{c}$ before the firing, the transfer block has no effect, on the other hand if the token was in $F_{c}$ then it is transfered to $T_{c}$.

Starting from the previous idea, we incrementally add new components to $M_{\varphi}$ so as to completely action of a global machine. An internal action a from state $p$ to state $q$ is simply modeled by adding an input arc from place $p$ and an output arc to place $q$ to the transition of the transfer (see Fig. B(b)). A rendezvous is treated similarly but with pairs of places (see Fig. B(c)). A broadcast sending is modelled as an internal action. Broadcast receptions are modeled via 
an unbounded transfer block that contains a transfer for each possible destination state (see Fig. 3(d)).

Finally, an asynchronous rendez-vous sending is modeled as an internal action, whereas the corresponding receptions are modeled via a transfer block with bound 1 that contains a transfer for each possible destination state (see Fig. 3(e)). A complete example treating a boolean formula and asynchronous rendez-vous is given in Fig. 3(f). The asynchronous rendez-vous update the boolean formula $b$ to false. The boolean part is modeled by an unbounded transfer block with one single transfer, the sender part by the petri net transition and the receivers part by a 1-bounded transfer block with two transfers. The following proposition formally relates global machines and MTNs.

Proposition 1. For any global machine $\mathcal{G}$ and any set of global states $\mathbf{G}$, we can construct automatically a MTN $\mathcal{M}_{\mathcal{G}}$ with only unbounded and 1-bounded multi-transfers such that $\alpha\left(\operatorname{Reach}_{\mathcal{G}}(\mathbf{G})\right)=\operatorname{Reach}_{\mathcal{M}_{\mathcal{G}}}(\alpha(\mathbf{G}))$.

\section{Verification of MTN-Based Abstract Models}

It is well-known that the class of safety properties of Petri Nets whose negation can be expressed in terms of upward closed sets of markings can be decided using backward reachability 19. In this setting the goal is to prove that none of the markings in a given infinite set $\mathbf{U}$ of unsafe configurations can be reached from the set of initial markings $\mathbf{M}_{0}$. To achieve this goal, we can first compute the transitive closure of the pre-image operator, and then check that no elements of $\mathbf{M}_{0}$ is in the resulting set of markings. As shown in [1,8,9], this algorithm is guaranteed to terminate for Petri Nets and Broadcast Protocols whenever $\mathbf{U}$ is upward closed w.r.t. the componentwise ordering of tuples. Formally, let cones $(\mathbf{S})=\left\{\mathbf{m}^{\prime} \mid \mathbf{m} \preccurlyeq \mathbf{m}^{\prime}, \mathbf{m} \in \mathbf{S}\right\}$. Then, a set of markings $\mathbf{S}$ is upward closed if cones $(\mathbf{S})=\mathbf{S}$. An upward closed set of markings $\mathbf{U}$ is always finitely generated by a set of minimal tuples, we will denote it as $\operatorname{gen}(\mathbf{U})$. The termination of the algorithm is ensured by the following properties. The application of the pre-image operator (associated to Petri Nets and Broadcast Protocols) to an upward closed set of markings returns a set that is still upward closed. The containment relation between upward closed sets of markings is a well-quasi ordering. It is important to note that Karp-Miller's construction may fail to terminate for extensions of Petri Nets with broadcast communication [8].

In order to extend the algorithm of [8], we first need to study the properties of the pre-image operator of MTNs.

Definition 10 (Pre-image of an MTN). Let $\mathcal{M}=\langle\mathcal{P}, \mathcal{B}\rangle$ be an MTN, and let $M \in \mathcal{B}$, then $\operatorname{Pre}_{M}(\mathbf{S})=\left\{\mathbf{m}^{\prime} \mid \mathbf{m}^{\prime} \succ_{M} \mathbf{m}, \mathbf{m} \in \mathbf{S}\right\}$.

We can easily prove that MTNs are monotonic with respect to the pointwise ordering on markings, i.e., if $\mathbf{m}_{1} \longmapsto \mathbf{m}_{2}$, then for any $\mathbf{m}_{1}^{\prime} \geq \mathbf{m}_{1}$ there exists $\mathbf{m}_{2}^{\prime} \geq \mathbf{m}_{2}$ such that $\mathbf{m}_{1}^{\prime} \longmapsto \mathbf{m}_{2}^{\prime}$.

Proposition 2. Let $\mathcal{M}=\langle\mathcal{P}, \mathcal{B}\rangle$ be an MTN, and $M \in \mathcal{B}$. If $\mathbf{S}$ is an upward closed set of markings, then $\operatorname{Pre}_{M}(\mathbf{S})$ is still upward closed. 
As a consequence, backward reachability for MTNs is guaranteed to terminate when taking an upward closed sets of markings as input. In the next section we will exploit the previous property to define a CST-based symbolic backward reachability for MTNs.

\section{The Assertional Language of Covering Sharing Trees}

Covering Sharing Trees (CSTs) are an extension of the sharing tree data structure introduced in [13] to efficiently store tuples of integers. A sharing tree $\mathcal{S}$ is a rooted acyclic graph with nodes partitioned in $k$-layers such that: all nodes of layer $i$ have successors in the layer $i+1$; a node cannot have two successors with the same label; finally, two nodes with the same label in the same layer do not have the same set of successors. Formally, $\mathcal{S}$ is a tuple $\left(N, V\right.$, root, end, val, succ), where $N=\{$ root $\} \cup N_{1} \cup \ldots \cup N_{k} \cup\{$ end $\}$ is the finite set of nodes $\left(N_{i}\right.$ is the set of nodes of layer $i$ and, by convention, $N_{0}=\{$ root $\}$ and $\left.N_{k+1}=\{e n d\}\right), V=\left\{x_{1}, x_{2}, \ldots, x_{k}\right\}$ is a set of variables. Intuitively, $N_{i}$ is

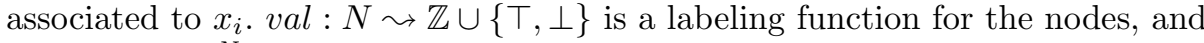
succ : $N \leadsto 2^{N}$ defines the successors of a node. Furthermore, $(1) \operatorname{val}(n)=\top$ iff $n=\operatorname{root}, \operatorname{val}(n)=\perp$ iff $n=$ end, $\operatorname{succ}($ end $)=\emptyset ;(2)$ for $i: 0, \ldots, k, \forall n \in N_{i}$, $\operatorname{succ}(n) \subseteq N_{i+1}$ and $\operatorname{succ}(n) \neq \emptyset ;(3) \forall n \in N, \forall n_{1}, n_{2} \in \operatorname{succ}(n)$, if $n_{1} \neq n_{2}$ then $\operatorname{val}\left(n_{1}\right) \neq \operatorname{val}\left(n_{2}\right)$. (4) $\forall i, 0 \leq i \leq k, \forall n_{1}, n_{2} \in N_{i}, n_{1} \neq n_{2}$, if $\operatorname{val}\left(n_{1}\right)=\operatorname{val}\left(n_{2}\right)$ then $\operatorname{succ}\left(n_{1}\right) \neq \operatorname{succ}\left(n_{2}\right)$. A path of a $k$-sharing tree is a sequence of nodes $\left\langle n_{1}, \ldots, n_{m}\right\rangle$ such that $n_{i+1} \in \operatorname{succ}\left(n_{i}\right)$ for $i=1, \ldots, m$-1. Paths represent $t u$ ples of size $k$ of natural numbers. We use $\operatorname{elem}(\mathcal{S})$ to denote the flat denotation of a $k$-sharing tree $\mathcal{S}$ :

$$
\operatorname{elem}(\mathcal{S})=\left\{\left\langle\operatorname{val}\left(n_{1}\right), \ldots, \operatorname{val}\left(n_{k}\right)\right\rangle \mid\left\langle\top, n_{1}, \ldots, n_{k}, \perp\right\rangle \text { is a path of } \mathcal{S}\right\} .
$$

Conditions (3) and (4) ensure the maximal sharing of prefixes and suffixes among the tuples of the flat denotation of a sharing tree. The size of a sharing tree is the number of its nodes and edges. The number of tuples in elem $(\mathcal{S})$ can be exponentially larger than the size of $\mathcal{S}$. As shown in [13], given a set of tuples $\mathcal{A}$ of size $k$, there exists a unique sharing tree such that $\operatorname{elem}\left(\mathcal{S}_{\mathcal{A}}\right)=\mathcal{A}$ (modulo isomorphisms of graphs). A CST is obtained by lifting the denotation of a sharing tree $\mathcal{S}$ as follows

$$
\operatorname{cones}(\mathcal{S})=\{\mathbf{m} \mid \mathbf{n} \preccurlyeq \mathbf{m}, \mathbf{n} \in \operatorname{elem}(\mathcal{S})\}
$$

$($ cone $(\mathbf{m})$ is defined in a similar way on a single marking $\mathbf{m})$. Given an upward closed set of markings $\mathbf{U}$, we define the CST $\mathcal{S}_{\mathbf{U}}$ as the $k$-sharing tree such that $\operatorname{elem}\left(\mathcal{S}_{\mathbf{U}}\right)=\operatorname{gen}(\mathbf{U})$. Thus, $\mathcal{S}_{\mathbf{U}}$ can be used to compactly represent gen $(\mathbf{U})$ (in the best case the size of $\mathcal{S}_{\mathbf{U}}$ is logarithmic in the size of gen $(\mathbf{U})$ ) and to finitely represent $\mathbf{U}$. A CST can also be viewed as a compact representation of the formula $\vee_{\mathbf{m} \in \operatorname{gen}(\mathbf{U})}\left(x_{1} \geq m_{1} \wedge \ldots \wedge x_{n} \geq m_{n}\right)$. An examples of CST is given in the of Fig. [5]a). 


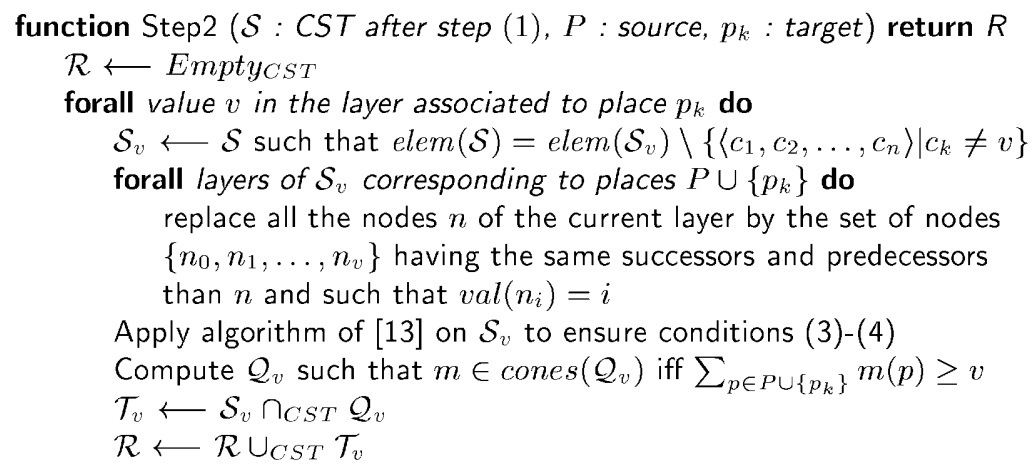

Fig. 4. Algorithm for Step (2).

\section{CST-Based Symbolic Pre Operator for MTNs}

In the context of Petri Nets, in [5], we presented an algorithm to symbolically compute the pre-image of a set of markings represented via a CST. In this section we will restrict ourselves to consider transfer blocks having bound $+\infty$. The algorithm can be extended however to any bound $c \in \mathbb{N}$, and in particular to $c=1$. Let us first note that the Pre operator enjoys the following properties.

Remark 1. Consider the multi-transfer $M=\left\langle T,\left\{S_{1}, \ldots, S_{k}\right\}\right\rangle$ where $T=\langle\mathcal{I}, \mathcal{O}\rangle$. We define $\operatorname{Pre}_{\mathcal{I}}, \operatorname{Pre}_{\mathcal{O}}$, and $\operatorname{Pre}_{S_{i}}$ as the pre-image operator associated to the multi-transfers $M_{\mathcal{I}}=\langle\langle\mathcal{I}, \emptyset\rangle, \emptyset\rangle, M_{\mathcal{O}}=\langle\langle\emptyset, \mathcal{O}\rangle, \emptyset\rangle$, and $M_{i}=\left\langle\langle\emptyset, \emptyset\rangle,\left\{S_{i}\right\}\right\rangle$, respectively. From Def. [6] we have that $\operatorname{Pre}_{M}=\operatorname{Pre}_{\mathcal{I}} \circ \operatorname{Pre}_{S_{1}} \circ \ldots \circ \operatorname{Pre}_{S_{k}} \circ$ Pre $_{\mathcal{O}}$. Furthermore, let $S$ be the transfer block $\left\langle\left\{B_{1}, \ldots, B_{r}\right\},+\infty\right\rangle$, then $\operatorname{Pre}_{S}=$ Pre $_{B_{1}} \circ \ldots \circ$ Pre $_{B_{r}}$, where Pre $B_{i}$ is associated to the multi-transfer $N_{i}=\left\langle T^{\prime},\left\{S_{i}^{\prime}\right\}\right\rangle$ such that $T^{\prime}=\langle\emptyset, \emptyset\rangle$ and $S_{i}^{\prime}=\left\langle B_{i}, c\right\rangle$. The previous properties hold because transfers are defined on distinct set of places each other.

Based on the previous remark, let us consider then a transfer block $M_{B}$ with bound $+\infty$ and with the single transfer $B=\left\langle P, p_{k}\right\rangle, P \subseteq \mathcal{P}$, and $p_{k} \in \mathcal{P}$. Furthermore, let $I_{P}=\left\{i \mid p_{i} \in P\right\}$ be the set of indexes of places in $P$. Given a $\operatorname{CST} \mathcal{S}$, our aim is to build an algorithm to construct a CST $\mathcal{S}^{\prime}$ such that $\operatorname{cones}\left(\mathcal{S}^{\prime}\right)=\operatorname{Pre}_{M_{B}}($ cones $(\mathcal{S}))$. We proceed in two steps.

The first step consists in removing all paths of $\mathcal{S}$ that do not satisfy the the post-condition induced by the semantics of transfer blocks with bound $+\infty$ : all source places in $P$ must contain zero tokens after firing $M_{B}$. Specifically, we compute the $\operatorname{CST} \mathcal{S}_{1}$ such that

$$
\operatorname{elem}\left(\mathcal{S}_{1}\right)=\left\{\left\langle c_{1}, c_{2}, \ldots, c_{n}\right\rangle \in \operatorname{elem}(\mathcal{S}) \mid c_{i}=0, \text { for any } i \in I_{P}\right\} .
$$

By construction, it follows that $\operatorname{Pre}_{M_{B}}\left(\operatorname{cones}\left(\mathcal{S}_{1}\right)\right)=\operatorname{Pre}_{M_{B}}(\operatorname{cones}(\mathcal{S}))$. This step can be performed in polynomial time in the size of $\mathcal{S}$ : we simply have to remove all nodes $n$ of the layers associated to places in $P$ such that $\operatorname{val}(n) \neq 0$. 
This will give us what is called a pre-Sharing Tree in 13, a graph in which condition (4) of the definition of sharing tree might be violated. By applying the algorithm described in [13, the pre-Sharing Tree can be re-arranged into a Sharing Tree in polynomial time.

As a second step, we compute the predecessors of the elements of $\mathcal{S}_{\mathbf{1}}$, w.r.t. $M_{B}$ (recall that $M_{B}$ has only the transfer $\left.B=\left\langle P, p_{k}\right\rangle\right)$. Suppose $\mathbf{c}=$ $\left\langle c_{1}, \ldots, c_{n}\right\rangle \in \operatorname{elem}\left(\mathcal{S}_{1}\right)$. Then, we know that the tuple c represents the upwardclosed set of markings $\mathbf{S}=\left\{\mathbf{m} \mid \mathbf{m}\left(p_{i}\right) \geq c_{i}, i: 1, \ldots, n\right\}$. Applying Pre $_{M_{B}}$ to $\mathbf{c}$, we should obtain a representation of the upward-closed set $\mathbf{S}^{\prime}$ whose markings present one possible distribution of tokens before the transfer from $P$ to $p_{k}$ took place. Note that the relation between the number of tokens in $P\left(\right.$ say $\left.\sum_{i \in I_{P}} x_{i}\right)$ and in $p_{k}\left(\right.$ say $\left.x_{k}\right)$ before and after firing $M_{B}$ is as follows: $x_{k}^{\prime}=x_{k}+\sum_{i \in I_{P}} x_{i}$ and $x_{i}^{\prime}=0$ for any $i \in I_{P}$. Thus, $\mathbf{S}^{\prime}$ will be generated by the set $\operatorname{gen}\left(\mathbf{S}^{\prime}\right)$ consisting of the markings $\mathbf{d}=\left\langle d_{1}, d_{2}, \ldots, d_{n}\right\rangle$ having the following properties: $d_{k}+\sum_{i \in I_{P}} d_{i}=c_{k}$; whereas $d_{j}=c_{j}$ for any $j \notin\left(I_{P} \cup\{k\}\right)$. Intuitively, all we need here is to forget about the labels of the nodes of $\mathcal{S}_{1}$ associated to the places in $P \cup\left\{p_{k}\right\}$, and replace them with nodes so that the sum of the values (associated to $P \cup\left\{p_{k}\right\}$ ) along a path always gives $c_{k}$.

For instance, consider a transfer $\left\langle\left\{p_{1}\right\}, p_{2}\right\rangle$, and let $c_{2}=2$ be the constant in the constraint associated to $p_{2}$ in $\mathcal{S}_{1}$. Furthermore, suppose that $p_{1}$ and $p_{2}$ are associated to adjacent layers in our CST. Then, we simply have to add the labels $0,1,2$ in both layers, and then connect the resulting nodes so that the sum of the connected values is always equal to 2 .

In the general case, we also have to take into account places stored in nonadjacent layers, consider more than one value for $c_{k}$, etc. To attack these problems, we split the algorithm in two sub-steps. For each possible value of $c_{k}$ in $\mathcal{S}_{1}$, we first compute an over-approximation (see the example below), and then, we select the exact paths by intersecting the resulting CST with a CST whose generators are the markings $\mathbf{m}=\left\langle m_{1}, \ldots, m_{n}\right\rangle$ in which $m_{k}+\sum_{i \in I_{P}} m_{i}=c_{k}$ and $m_{j}=0$ for $j \notin I_{P} \cup\{k\}$. The complete algorithm is given in Fig. 4 . The following example will help in understanding this technique.

As an example, consider the CST $\mathcal{S}$ in Fig. 5(a) consisting of the three elements $\langle 0,2,0,1,0,0\rangle,\langle 0,1,0,2,0,0\rangle$ and $\langle 0,0,0,2,2,0\rangle$ representing the formula $\Phi=\left(c_{2} \geq 2 \wedge c_{4} \geq 1\right) \vee\left(c_{2} \geq 1 \wedge c_{4} \geq 2\right) \vee\left(c_{4} \geq 2 \wedge c_{5} \geq 2\right)$. Now consider the transfer that moves the tokens from place $p_{5}$ into place $p_{2}$, defined through the equation $c_{2}^{\prime}=c_{2}+c_{5}, c_{5}^{\prime}=0$. When applied to the CST (a) the algorithm of Fig. 4 performs the steps shown in (b-g). Specifically, it first computes (b) by removing the tuple $\langle 0,0,0,2,2,0\rangle$ that do not satisfy $c_{5}^{\prime}=0$. At the second iteration of the loop (line 3, Fig 4), it computes (c). By adding new nodes in the second and fifth layers, we obtain (d), an over-approximation of the backward reachable markings starting from (c). The $\operatorname{CST} \mathcal{Q}_{2}$ representing all the tuples satisfying $c_{2}+c_{5} \geq 2$ is shown in (e). The CST resulting from the intersection of (d) and $\mathcal{Q}_{2}$ corresponding to the exact set of backward reachable markings starting from (c) is shown in (f). Finally, the result of the algorithm is the CST (g). 


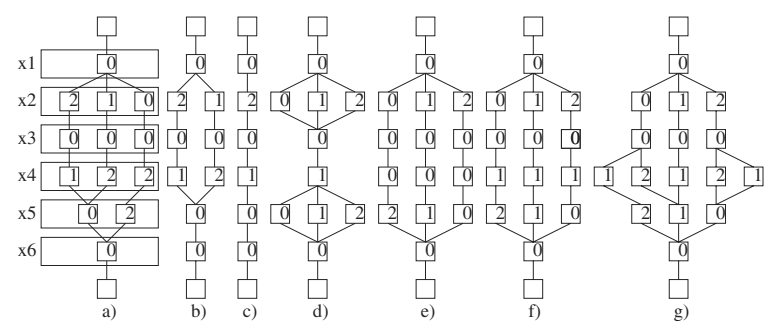

Fig. 5. Set of CST generated during the computation of the transfer $c_{2}^{\prime}=c_{2}+c_{5}, c_{5}^{\prime}=0$

Structural Heuristics. The MTNs that results from the translations from global machines enjoy the following interesting property. They can be viewed as Petri Nets with arc dependent cardinality arcs [3], an extension of Petri Nets in which edges are labeled with functions linear in the current marking. Ciardo has shown that place invariants for Petri Nets with arc dependent cardinality arcs can be computed by first reducing them to Petri Nets (Theorem 1 of [3]). When specialized to a given initial marking, place invariants can be used to infer structural invariants that must hold in all reachable markings (i.e. they represent an overapproximation of the reachability set). Structural invariants can then be used to prune the search space explored during backward reachability. On the basis of the previous observation, we can enhance the MTN-backward reachability algorithm with the efficient pruning techniques working on CSTs we proposed in 6]. In this technique we use the information coming from the statically computed invariants as follows. At each step during the search we remove paths of the CST representing the partial search space that do not satisfy the invariant. This operations can be efficiently performed by working directly on the structure of a CST. In the next section we will report on practical experiments we obtained with the resulting method.

\section{$7 \quad$ Experimental Results}

The table in Fig. 6 describes some of the results we obtained by applying the extended CSTs-library to examples of MTNs modeling multithreaded programs. As an example, we have tested the MTN corresponding to the global machine partially described in Fig. 2 We considered the following unsafe set of states: at least two threads have modified only one of the two coordinates of the Point object. This upward closed set of states can be represented by CSTs in which either at least two tokens are in the place corresponding to the incY() state of Fig. 2, or at least one token is in state $\operatorname{incX()}$ and at least one token is in state $\operatorname{dec}()$, or at least two tokens are in state $\operatorname{decY}()$. For this example, we have automatically computed place invariants, and we have applied them to prune the search as shown in Fig. 6(I/D). Here $m$ and $n$ represent the number of Inc and Dec threads in the initial marking. We considered both parametric initial markings (e.g. $m \geq 1, n \geq 1$ ) as well as fixed initial markings (e.g. $m=1, n=1$ ). 


\begin{tabular}{|l|c|c|c|c|c|r|r|r|r|r|r|}
\hline MTN & $\mathbf{m}_{0}$ & $\mathbf{P}$ & $\mathbf{M T}$ & $\mathbf{I}$ & $\mathbf{E r r}$ & $\mathbf{S}$ & $\mathbf{N N}$ & $\mathbf{N E}$ & $\mathbf{E T C}$ & $\mathbf{E T H}$ & $\mathbf{R}$ \\
\hline \hline $\mathrm{I} / \mathrm{D}$ & $m, n \geq 1$ & 32 & 28 & & $\sqrt{ }$ & 10 & 1542 & 1823 & $9.77 \mathrm{~s}$ & $\uparrow$ & - \\
\hline $\mathrm{I} / \mathrm{D}$ & $m, n \geq 1$ & 32 & 28 & $\sqrt{ }$ & $\sqrt{ }$ & 10 & 538 & 209 & $0.82 \mathrm{~s}$ & $40.24 \mathrm{~s}$ & 49 \\
\hline $\mathrm{I} / \mathrm{D}$ & $m, n=1$ & 32 & 28 & $\sqrt{ }$ & $\sqrt{ }$ & 10 & 279 & 68 & $0.22 \mathrm{~s}$ & $5.07 \mathrm{~s}$ & 23 \\
\hline $\mathrm{P} / \mathrm{C}$ & $m, n=2$ & 18 & 14 & & & 25 & 860 & 12264 & $44.49 \mathrm{~s}$ & $\uparrow$ & - \\
\hline $\mathrm{P} / \mathrm{C}$ & $m, n=2$ & 18 & 14 & $\sqrt{ }$ & & 8 & 111 & 43 & $0.04 \mathrm{~s}$ & $0.46 \mathrm{~s}$ & 11.5 \\
\hline $\mathrm{P} / \mathrm{C}$ & $m, n=50$ & 18 & 14 & $\sqrt{ }$ & & 152 & 8511 & 179451 & $1 \mathrm{~h} 36 \mathrm{~m}$ & $\uparrow$ & - \\
\hline $\mathrm{P} / \mathrm{C}_{1}$ & $k, l, m, n \geq 1$ & 44 & 37 & & $\sqrt{ }$ & 14 & 20421 & 15543 & $37 \mathrm{~m} 55 \mathrm{~s}$ & $\uparrow$ & - \\
\hline $\mathrm{P} / \mathrm{C}_{1}$ & $k, l, m, n \geq 1$ & 44 & 37 & $\sqrt{ }$ & $\sqrt{ }$ & 14 & 1710 & 1384 & $2.86 \mathrm{~s}$ & $\uparrow$ & - \\
\hline $\mathrm{P} / \mathrm{C}_{1}$ & $k, l, m, n=1$ & 44 & 37 & $\sqrt{ }$ & $\sqrt{ }$ & 14 & 1231 & 736 & $1.88 \mathrm{~s}$ & $\uparrow$ & - \\
\hline $\mathrm{P} / \mathrm{C}_{2}$ & $k, l, m, n \geq 1$ & 44 & 37 & & & 29 & 12479 & 8396 & $55 \mathrm{~m} 50 \mathrm{~s}$ & $\uparrow$ & - \\
\hline $\mathrm{P} / \mathrm{C}_{2}$ & $k, l, m, n \geq 1$ & 44 & 37 & $\sqrt{ }$ & & 1 & 46 & 1 & $0.02 \mathrm{~s}$ & $1.73 \mathrm{~s}$ & 86.5 \\
\hline $\mathrm{P} / \mathrm{C}_{2}$ & $k, l, m, n=1$ & 44 & 37 & $\sqrt{ }$ & & 1 & 46 & 1 & $0.00 \mathrm{~s}$ & $0.48 \mathrm{~s}$ & $>48$ \\
\hline \hline
\end{tabular}

Fig. 6. Benchmarks on an AMD Athlon 900Mhz 500Mbytes: $\mathbf{m}_{0}=$ initial marking, $\mathbf{P}=\mathrm{n}$. places, $\mathbf{M T}=$ n. multi-transfers; $\mathbf{I}=$ pruning via invariants; $\mathbf{E r r}=$ bug found; $\mathbf{S}=$ n. iterations before reaching the fixpoint/finding a bug; $\mathbf{N N}=$ nodes of the CST-fixpoint; $\mathbf{N E}=$ paths in the CST-fixpoint; ETC=ex. time using CSTs; $\mathbf{E T H}=$ ex. time using HyTech ( $\uparrow$ indicates that HyTech ran out of memory); $\mathbf{R}=\mathbf{E T H} / \mathbf{E T C}$.

In the second case the idea of pruning the search space via structural invariants is much more effective (see [6]). In all cases our tool found a potential bug (see Fig, (6), that (after looking at the abstract trace) turns out to be a mistake in the Java program. In fact, though the primitive methods incx, incy, decx, and decy are protected by a monitor (they are declared as synchronized), the derived methods incpoint and decpoint are not. Thus, increments(decrements) on pair of coordinates are not executed atomically. To correct the error, we can declare the derived methods synchronized, too. This way mutual exclusion is automatically guaranteed by the semantics of Java.

We have also analyzed the Producer-Consumer example of [4] $(\mathrm{P} / \mathrm{C}$ in Fig. 6) and a modified version of it $\left(\mathrm{P} / \mathrm{C}_{1}\right.$ in Fig. 6) built as follows: We introduced new class declarations for malicious producers and consumers, and we artifically inserted the possibility of violating mutual exclusion. In this example the presence of violations depends on the values of boolean variables. Our tool finds the bug after 14 iterations (see Fig. 6). We also managed to verify the corrected version in less than one second $\left(\mathrm{P} / \mathrm{C}_{2}\right.$ in Fig. 6). As shown in Fig. 6. we ran the same examples (using the same invariants) on HyTech [11]. Our execution times are always better. Furthermore, in some case HyTech ran out of memory before reaching a fixpoint or detecting the presence of the initial state.

\section{Conclusions and Related Works}

In this work we focused on the following points. Via a connection with previous works on parameterized verification [7]8], we have shown that there exists an extension of Petri Nets that captures the essential features of the concurrent model of Java. For this class, we can use decision procedures to automatically 
verify safety properties for arbitrary number of threads. This goal is achieved by extending the CST-based symbolic model checking algorithm previously defined for Petri Nets.

The use of parameterized verification via backward reachability is the main novelty of this approach over other approaches to software verification via finite models (see e.g. [4]) or with Petri Nets like model [2]. The verification approach of [2] is based on the Karp-Miller's coverability tree that amount to forward reachability with accelerations (see also [7]). Contrary to backward reachability, the automated construction of Karp-Miller coverability tree is not guaranteed to terminate for extensions of Petri Nets with transfer arcs as shown by the counterexample of Esparza, Finkel, and Mayr [8].

Our preliminary analysis of the problem, tune of the techniques, and experimental results indicate the potential interest of a second research phase aimed at producing automatically infinite-state skeletons of Java programs, a task that is in an advanced stage in the finite-case verification approach. This will be one of our main future directions of research. As we explain in the paper, our techniques find other interesting applications for the automated verification of Broadcast Protocols. Concerning this point, we are not aware of other tools designed to attack symbolic state explosion for this class of extended Petri Nets.

\section{References}

1. P. A. Abdulla, K. Cerāns, B. Jonsson and Y.-K. Tsay. General Decidability Theorems for Infinite-State Systems. In Proc. LICS'96, pages 313-321, 1996.

2. T. Ball, S. Chaki, S. K. Rajamani. Parameterized Verification of Multithreaded Software Libraries. In Proc. TACAS'01, LNCS 2031, pages 158-173, 2001.

3. G. Ciardo. Petri Nets with marking-dependent arc multiplicity: properties and analysis. In Proc. ICATPN'94, LNCS 815, pages 179-198, 1994.

4. J. C. Corbett. Constructing Compact Models of Concurrent Java Programs. In Proc. ISSTA'98, pages 1-10, 1998.

5. G. Delzanno, and J.-F. Raskin. Symbolic Representation of Upward Closed Sets. In Proc. TACAS 2000, LNCS 1785, pages 426-440, 2000.

6. G. Delzanno, J.-F. Raskin, and L. Van Begin. Attacking Symbolic State Explosion. In Proc. CAV'01, LNCS 2102, pages 298-310, 2001.

7. E. A. Emerson and K. S. Namjoshi. On Model Checking for Non-deterministic Infinite-state Systems. In Proc. of LICS '98), pages 70-80, 1998.

8. J. Esparza, A. Finkel, and R. Mayr. On the Verification of Broadcast Protocols. In Proc. LICS'99, pages 352-359, 1999.

9. A. Finkel and P. Schnoebelen. Well-structured transition systems everywhere! TCS 256 (1-2):63-92, 2001.

10. S. M. German, A. P. Sistla. Reasoning about Systems with Many Processes. JACM 39(3): 675-735 (1992)

11. T. A. Henzinger, P.-H. Ho, and H. Wong-Toi. НyTEch: a Model Checker for Hybrid Systems. In Proc. CAV'97, LNCS 1254, pages 460-463, 1997.

12. D. Lea. Concurrent Programming in Java. Design Principle and Patterns. Second Edition. The Java Series. Addison Wesley, 2000.

13. D. Zampuniéris, and B. Le Charlier. Efficient Handling of Large Sets of Tuples with Sharing Trees. In Proc. DCC'95, 1995. 